\title{
Distribution of stereoanomalies in the general population
}

\author{
MILLICENT NEWHOUSE and WILLIAM R. UTTAL \\ Perception Laboratory, Institute for Social Research, University of Michigan \\ Ann Arbor, Michigan 48106
}

\begin{abstract}
Large proportions of the general population have frequently been reported to be stereoscopically anomalous. However, when we tested a large sample (103 subjects), we found all (with the exception of three truly stereoblind observers) to be able to initially detect depth in Julesz random dot stereograms within $2 \mathrm{~min}$. Some subjects, however, were not able to detect depth when retested immediately with reversed disparity, but half of those were able to see depth on retesting a few days later. We conclude that stereoanomalies are much rarer than has previously been suggested and that any putative one-way (i.e., restricted to crossed or uncrossed disparity) deficiencies are actually due to strategy or sequence effects rather than to neural deficiencies.
\end{abstract}

Stereopsis-depth perception cued by disparity differences between the images projected on the two retinae by a single object-has been reported to be frequently anomalous in the general population. Richards (1970), for example, has reported that as much as $30 \%$ of the general population may suffer from at least a partial form of stereoanomaly. Similarly, Jones (1977) found six cases of stereoanomaly in 30 subjects. However, in the search for subjects in experiments carried out by our laboratory, our experience has been that most candidates pass informal screening tests for stereopsis. We, therefore, set out to determine if the previously reported high levels of stereoanomaly were valid by surveying a larger sample of the general population in a controlled experiment. The results of our survey indicate that, if care is given to the order of testing, most initially suspect stereoanomalies disappear and that stereoscopic vision is deficient in only a very small proportion (less than $6 \%$ ) of the population. This conclusion has also been recently drawn by Patterson and Fox (Note 1). We all agree that previous reports of high levels of stereoanomaly in the general population are due to uncontrolled sequence effects and that the actual rate of occurrence is much lower than has been previously reported.

\section{METHOD}

\section{Subjects}

One hundred and two ( 33 male and 69 female) randomly selected employees of the University of Michigan Institute for

This research was supported by Office of Naval Research Contract N00014-81-C-0266 to the University of Michigan. Millicent Newhouse is the current (1981-1982) ONR Science Apprentice in the ISR Perception Lab; she is a senior at Ann Arbor Huron High School.
Social Research volunteered to serve for a single test session lasting approximately $10 \mathrm{~min}$.

\section{Procedure}

The purpose of the experiment was explained to each participant, and a data sheet asking general biographical questions was compiled. The subjects were then shown a series of anaglyphic random dot stereograms (Figure 1.0-1, a triangle; Figure 2.4-1, a square; and Figure 8.1-2, a series of squares with decreasing correlation; all of which were taken from Julesz, 1971). The first two stereograms are samples designed to teach the general method. Following this pretraining with these two highcorrelation samples, the series of seven stereograms with decreasing proportions of correlated dots $(100 \%, 90 \%, 80 \%, 70 \%$, $60 \%, 50 \%, 40 \%$ ) was presented in order. The time it took for the subject to report the appearance of depth was measured for each stereogram with a stopwatch.

Each subject was given a maximum of $2 \mathrm{~min}$ in which to detect depth for each picture. When the subject reported depth, the time was recorded and the subject was asked to describe what was seen. If the description of the form was correct on the two sample forms, the series of stereograms with decreasing correlation was presented. Subjects were timed for each stereogram in the series either until the $40 \%$ minimum correlation stereogram had been presented or until a stereogram was presented for which they were unable to report depth in the allotted 2 -min period. The subject was then asked to reverse the redgreen lenses (so that the red filter was positioned in front of the left eye and the green filter in front of the right eye) and was then retested with the decreasing-correlation series. This was done to determine if the response to either crossed or uncrossed disparity was deficient, those disparity conditions being reversed when the lenses were exchanged in front of each eye.

\section{RESULTS}

Of the 102 subjects, all but 3 initially reported depth in the two sample (triangle and square) stereograms. The point at which depth was no longer perceived in the series of stereograms with decreasing correlation varied. Decreasing proportions of the survey group reported depth as the stereograms were presented in order of 
decreasing correlation. These data are summarized in Figure 1.

When the colored lenses were reversed, three male and nine female subjects were initially unable to detect the pattern. To determine if this finding was due to a bias or sequence effect, we retested each of these 12 subjects with the two sample patterns several days later with the lenses initially in the reversed condition. Six of the 12 subjects did report depth and identified the sample pattern correctly in the retest. The other six (four female and two male) did not correctly identify the patterns in the allowed 2-min period.

We also measured the time it took each subject to report depth in the two sample stereograms up to the 2 -min maximum. Figure 2 is a graph showing the proportion of the group reporting depth at the indicated times. As indicated in this graph, half of the subjects reported depth in $5 \mathrm{sec}$ or less and virtually all in $25 \mathrm{sec}$ or less. Beyond $25 \mathrm{sec}$, all points plotted are for only one or two subjects. Figure 3 plots the same information for the series of stereograms with decreasing correlation. Obviously, there is a progressive prolongation of the time taken by the group to detect a stereogram as the correlation decreases. This graph shows that $61 \%$ of the people saw the first stereogram in the series $(100 \%)$, a little over $32 \%$ of the participants identified the $90 \%$ and $80 \%, 13 \%$ saw the $70 \%$, and $19 \%$ saw the $60 \%$

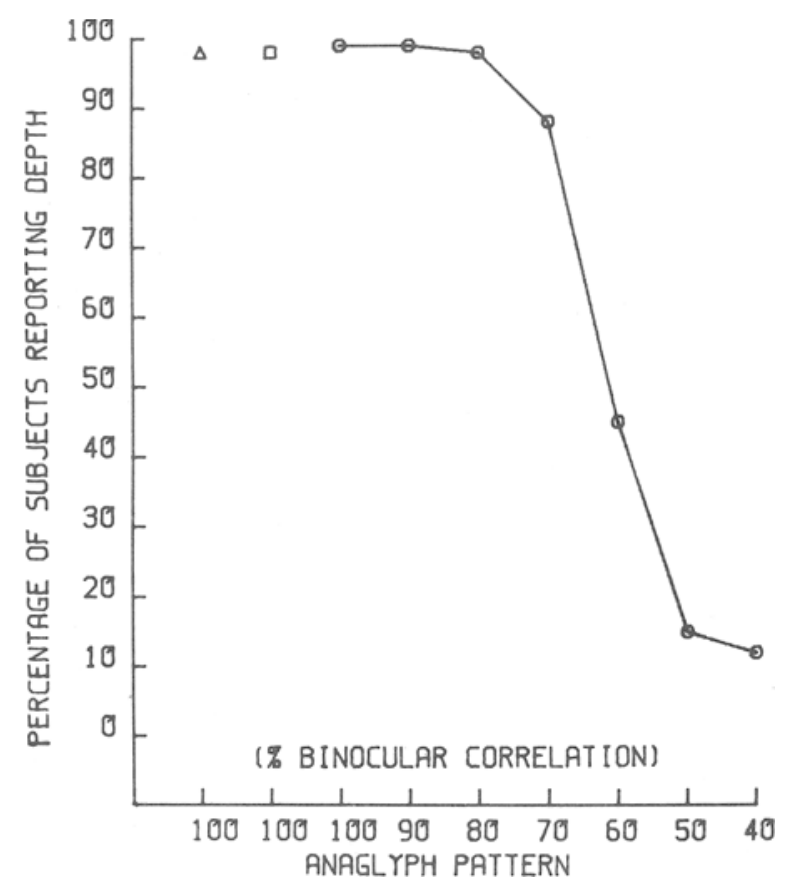

Figure 1. Percentage of subjects reporting depth in less than $2 \mathrm{~min}$ as a function of the binocular correlation of random dot stereograms. $\Delta$ indicates a triangular sample stimulus pattern, $\square$ indicates a square sample stimulus pattern, and the circles indicate a series of square stimulus patterns with decreasing binocular correlation.

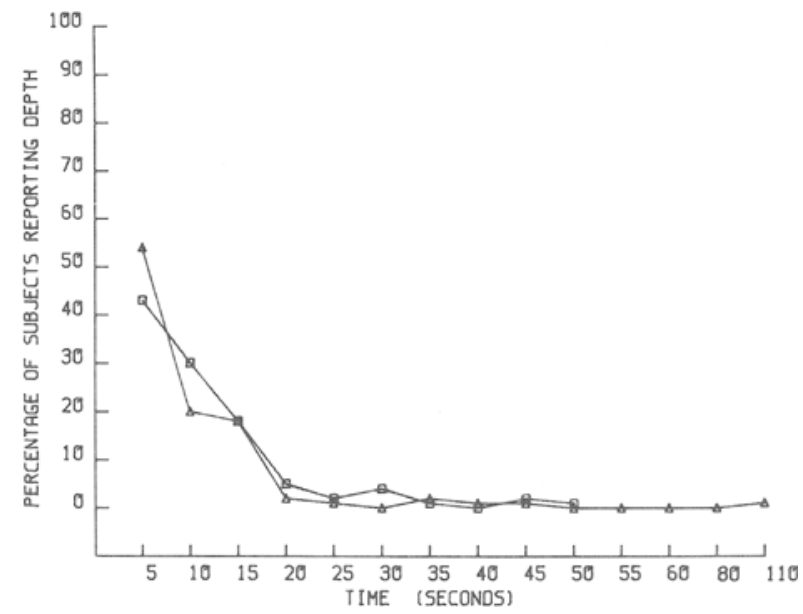

Figure 2. The percentage of subjects reporting depth for the two sample stereograms (triangle and square) as a function of observation time.

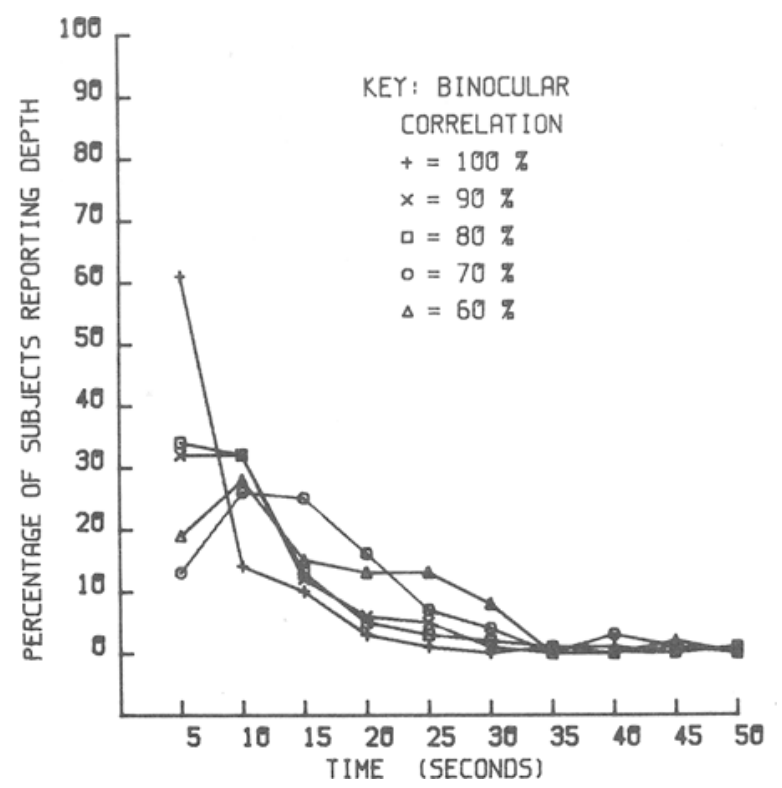

Figure 3. The percentage of subjects reporting depth for the series of decreasingly correlated stereograms as a function of time.

stereograms in less than 5 sec. In general, the lower the correlation, the longer it took to perceive depth.

\section{CONCLUSION}

Our results indicate that the distribution of total stereoblindness is smaller than has been previously reported. Only three of our subjects were totally stereoblind. Of these three, one, it turned out, had only one real eye; one had a history of surgically corrected congenital strabismus, and only one was visually normal in all other regards (as well as quite surprised to discover that she suffered from this anomaly). The small number of subjects who were determined to display stereoanomaly in either crossed or uncrossed direction (but not both) was also 
smaller than has been previously reported. Most important, given that all except the three stereoblind subjects initially saw depth in the sample pattern, we conclude that one-way stereoanomaly is more likely to be a sequence effect than the result of a neural deficiency.

\section{REFERENCE NOTE}

1. Patterson, R. E., \& Fox, R. Information processing in global stereopsis. Paper presented at the annual meeting of the Psychonomic Society, Philadelphia, November 1982.

\section{REFERENCES}

Jones, R. Anomalies of disparity detection in the human visual system. Journal of Physiology, 1977, 264, 621-640.

Julesz, B. Foundations of cyclopean perception. Chicago: University of Chicago Press, 1971.

RichaRDs, W. Stereopsis and stereoblindness. Experimental Brain Research, 1970, 10, 380-388.

(Received for publication May 23, 1982.) 